\title{
RaTTy: Mouse cum Pen
}

\author{
Sanjeev Gangwar \\ Assistant Professor \\ Department of Computer Applications \\ VBS Purvanchal University Jaunpur
}

\author{
Shubhranshu Tiwari \\ Department of Information Technology \\ VBS Purvanchal University \\ Jaunpur
}

\begin{abstract}
The name 'RaTTy' comes from the word 'Rat' which means 'Mouse' and the reason that it has been named as RaTTy is, the proposed technology is an enhancement of existing optical mouse and pen technology. RaTTy is an accurate combination of optical mouse and pen as the mouse structure has been reshaped and then a pen mechanism has been added up to make it similar to a 'Mouse cum Pen'.

RaTTy is a very cost effective and affordable product. It is a complete package of software and hardware. The software that comes with RaTTy provides user interface and other controlling functionality. The software also provides a tutorial feature that makes technical study very easy. It can bring a revolution in the optical mouse market.
\end{abstract}

\section{Keywords}

The main keywords are; RaTTy, RCS, Up-Down mechanism (UDM), finger and thumb button, scrolling hat, etc.

\section{INTRODUCTION}

The optical mouse has been a key and easily usable device to interact with computers because the shape of the device makes its users comfortable. On the other hand a pen is also a handy device to write on paper with control. In RaTTy technology, both of the above technologies have been embedded. Mouse can be used only for the specified purposes: clicking, dragging, scrolling, etc. but it becomes difficult to draw an object on 'Paint' like application. The device image has been shown below.

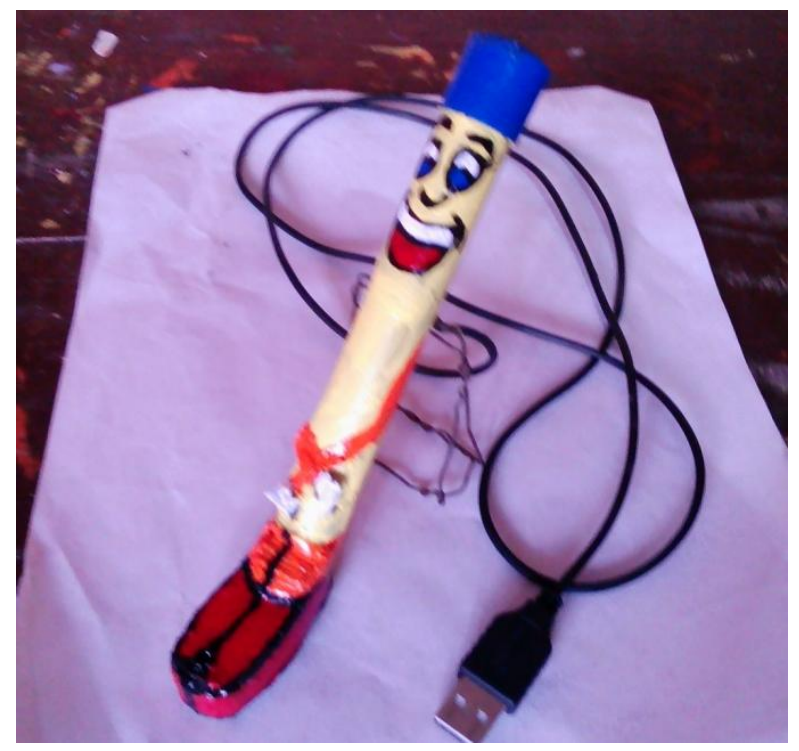

Fig 1: RaTTy Image

This device can be connected to the laptop's USB port without any extra requirement. It does not need any device driver other than the mouse default embedded driver, because the device main circuit is the mouse circuit and the software that may come within the package provides all the remaining features.

\section{RESEARCH BACKGROUND}

The technology that motivated research on this technology is optical mouse technology. And thus the study of the optical mouse has been the background of this research. The background research elaborated the reasons that a mouse cannot be used to draw objects smoothly. There are main two reason behind this. First reason is, the bottom surface area of the mouse is very large compared to pen pointer. Hence it provides a huge friction and when we move mouse on the surface we have to apply a small force which we have not to apply while writing with pen. Since we apply force to draw shapes, we couldn't draw them with control. Second reason is; to draw something, we need to press left button and then drag the mouse pointer, but in case of pen we don't need to do it. This extra burden makes a difference. Hence it becomes uncomfortable for the writer to write something with the mouse. Both reasons have been worked on in the proposed technology so that it can be a substitute product.

\section{TECHNOLGY OVERVIEW}

\subsection{Physical Structure}

The pen's height is $12.3 \mathrm{~cm}$ and width is $1.6 \mathrm{~cm}$. The bottom box that has contained sensor and LED has length $5.4 \mathrm{~cm}$ and width $1.6 \mathrm{~cm}$. The bottom box has been joined with the vertical cylinder at such an angle that while holding the pen, the box remains in horizontal plane, parallel to the surface. At the bottom side of the box, there is a triangle shape hole through which red light passes. The vertical cylinder contains two main button called as; finger and thumb button, work in similar manner to the mouse left and right button works respectively. The middle button is placed in the middle of the pen's body for the special dedicated purpose. At the top of the pen, there is a scrolling hat.

The pen has a special two way switch at its right side. This switch is used to decide whether the device would work as pen or as a traditional mouse. The pen may be of two type; wired pen (as shown in figure) and wireless pen. The wired pen has a USB cable and the wireless pen has a Bluetooth 2.0 which is used to connect it to the laptop.

\subsection{Up-Down Mechanism (UDM)}

The extra component that has been added to the mouse circuit is an Up-Down Mechanism which has been fixed at the bottom face of the horizontal box. This mechanism is a solution of the second reason specified under the above heading 'Research Background'. The principle of this mechanism is very similar to the principle of pen. According to which, the pen writes on the paper when the pointer touches the surface, and stops writing when the pointer gets back into the air (leaves the contact with the surface). To simulate this behavior, a pin component has been fixed just beside the 
sensor and named as Up-Down Mechanism (UDM). The conceptual view of the UDM in two cases: First when it's pushed (circuit gets complete i.e. finger button is pressed), and Second when it's in the air (Circuit gets broken), is shown in the following image.

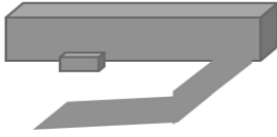

Before Pushing

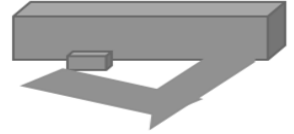

After Pushing
Fig 2: UDM

The logic is very simple and has been shown in the following table.

Table 1. Logic of UDM

\begin{tabular}{|c|c|c|c|}
\hline $\begin{array}{c}\text { In case of ordinary } \\
\text { pen }\end{array}$ & $\begin{array}{c}\text { ink comes } \\
\text { out }\end{array}$ & $\begin{array}{c}\text { In case of } \\
\text { UDM }\end{array}$ & $\begin{array}{c}\text { Circuit } \\
\text { Complete }\end{array}$ \\
\hline Pointer is grounded & Yes & Pin pushed & Yes \\
\hline Pointer is in the air & No & Pin in air & No \\
\hline
\end{tabular}

\subsection{Two-Way Switch}

To control the behavior of the device i.e. to decide whether it would work as a pen or as a mouse, there is given a two-way switch on the right most part of the vertical cylinder. The switch position decides the mode of the device. If the switch is pushed forward, the device becomes pen. That is, it shows following two changes

The mouse physical movements gets synchronized with the cursor movement and hence allows the writing mechanism. The writing mechanism is done in the same manner as we write on paint like application i.e. wherever the cursor moves, the shape is drawn. If the cursor is moved in a specific pattern making an alphabet shape, the alphabet is written on the screen.

The second thing that the forward pushing does is, to enable UDM and disable finger click. Hence the control of clicking the right button while writing is passed to the UDM and the writer does not need to click and hold the finger button. When the device pointer is grounded, the UDM goes into the pressed mode and behaves like the finger button is clicked. When the pointer gets back to the air, the UDM releases the button.

The second mode of the device is the mouse mode and is obtained by pushing the two way switch in backward direction. If the switch is pushed backward, the following two changes happen.

First, the cursor movement gets free i.e. the device comes back into normal operational mode and the cursor movement is not strictly controlled by the device physical movement.

The UDM is disabled and the finger button is enabled for mouse left clicking.

The implementation of this behavior is done by connecting the IC pin of finger button (or left button in case of mouse) with the middle pin of the switch and then connecting the left pin of the switch to the UDM and right pin of the switch to the finger button. Hence the two way switch now can decide in which device to send the signal coming from the IC in order to enable that particular device.

We also need to add a module in the controlling software which will accept the signal of mode from the two way switch. The existing mouse driver software should also be updated with the two way switch mechanism, so that when the device mode is selected as 'Pen', an instruction is sent to the computer software for extra arrangement. This arrangement may include the synchronization of the pen's actual physical movement to the computer's cursor movement so that it can bring a realistic experience as we get in case of traditional pen.

\subsection{RCS}

RaTTy Controlling Software (RCS) comes in the package with the hardware to make user interface of the device comfortable and interactive. This is a dedicated software that performs all the controlling activity of the device. The software main works are;

To sync the cursor speed with physical movement.

To provide all the basic tools that a writer need during writing as well as a painter need to make a painting.

To provide a tutorial facility which has been discussed later in the paper.

To accept the mode instruction from the two way switch.

To enable the users to prepare their word document. This feature allows to prepare word document by just writing on the simple sheet. After writing, the algorithm will scan every shape and prepare a digital data corresponding to drawn shapes by OCR methods. And hence the drawn curves will be converted into digital data. For example, if we draw a shape similar to ' $A$ ', the OCR method will store the shape ' $A$ ' in digital format so that it could be later on modified by the existing software.

\subsection{Tutorial Facility}

The main goal for making this pen is to make the class time education easier for technical students. The device can be used in technical education. To elaborate a technical topic and to make the students understand this topic clearly, the topic should be clearly depicted and the relevant figures should be drawn. For this purpose, a lecturer uses a laptop with a projector. He draws diagrams and shows technical picture to explain the topic.

This complete package may replace such requirements for this kind of technical education classes. The reason is explained below.

Suppose, the lecturer has this pen and a laptop that has RCS installed. In this software, the user gets a facility to share the RCS homepage screen on which he is writing with all those laptops which are connected to the same network through which the lecturer's laptop is connected. Every student who wants to study in the class room, may have a laptop having RCS installed and connected to the same network. He gets a facility to view the RCS homepage screen of lecturer's laptop live. He seems it like a video has been played on his laptop screen. The student gets a chance to make a small note by typing while the lecture is going on. After getting done, the student can download the complete video along with the notes that he made during lecture. The notes will, later on, appear as subtitle of a video. Hence he can relive the class room lecture later.

With such kind of facility, it becomes very easy and convenient for the students to study and to understand technical topics easily. 


\section{CIRCUIT DESIGN}

This is an enhancement of optical mouse circuit by the use of pen basic mechanism. The optical mouse circuit has been customized and fitted along a vertical line inside a vertical cylinder.

There are three main circuits in the device. Every circuit has their specific position and purpose. The components are;

\section{B. Vertical Circuit (VC) \\ C. Up-Down Mechanism Circuit (UDMC)}

The following picture explains every circuit and their connection. Horizontal circuit is in the top left corner. The VC is in right part and the UDM is in left bottom corner.

A. Horizontal Circuit (HC)

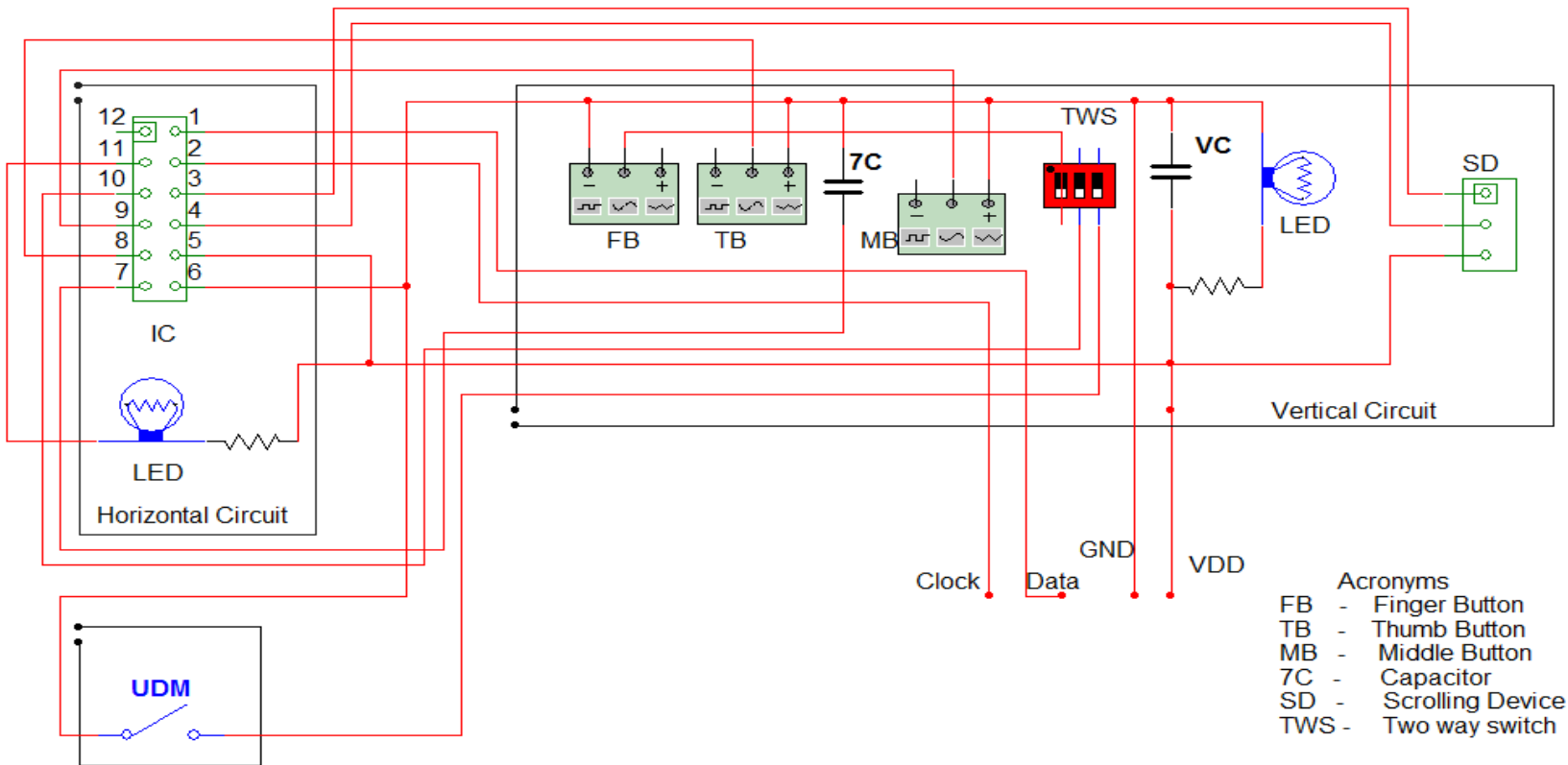

Fig 3: Circuit Diagram of RaTTy

\subsection{Horizontal Circuit (HC)}

The HC consists of only three components; 12 pin IC, down facing LED and resistor. IC has an embedded surface on its bottom portion.

\subsubsection{IC}

There are total 12 pins in the IC. These pins are connected to various other components of VC and UDMC. Following table shows the meaning of every pin of IC.

\begin{tabular}{|c|c|c|}
\hline Pin & Connect to & Description \\
\hline 1 & $\begin{array}{l}\text { Data wire of } \\
\text { USB cable }\end{array}$ & Transfers signal to computer \\
\hline 2 & $\begin{array}{c}\text { Clock wire of } \\
\text { USB }\end{array}$ & Gives clock signal to computer \\
\hline 3 & $\begin{array}{l}\text { Scrolling } \\
\text { Device }\end{array}$ & $\begin{array}{l}\text { Signal to Scrolling device } \\
\text { bottom pin }\end{array}$ \\
\hline 4 & $\begin{array}{l}\text { Scrolling } \\
\text { Device }\end{array}$ & $\begin{array}{l}\text { Signal to Scrolling device } \\
\text { middle pin }\end{array}$ \\
\hline 5 & VDD & Voltage wire of USB cable \\
\hline 6 & GND & Ground wire of USB Cable \\
\hline 7 & 7C & Capacitor \\
\hline 8 & Thumb Button & Sends right click signal \\
\hline 9 & Middle Button & Sends scroll fix signal \\
\hline 10 & Finger Button & Sends left click signal \\
\hline 11 & LED & Negative pin of LED \\
\hline
\end{tabular}

\subsubsection{LED \& Lens}

There is a red light LED which emerges a sharp spot of red light on the small surface area under the sensor. The LED is focused parallel to the horizontal circuit but the spot light is required on the surface. Hence the light beam needs to be bent. The light beam is bent by a triangle shape prism. The prism bends the beam at a particular angle so that it can incident on the small surface area and be reflected by that area into the down facing sensor.

\subsubsection{Sensor}

The sensor takes the snapshots of the small spot at a very rapid rate. It analyzes two consecutive snapshots to determine the changes in the pixel and hence decides in which direction the device has been moved. The following picture explains the concept.

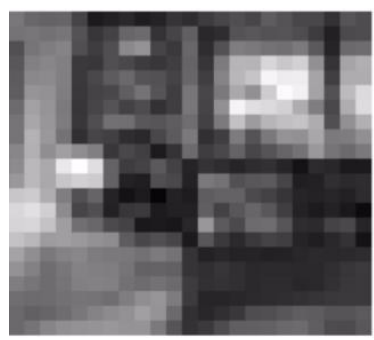

Image at $\mathrm{t}=0$ milisecond

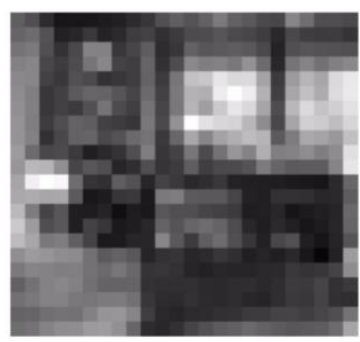

Image at $\mathrm{t}=0.2$ milisecond
Fig 4: Snapshots at a Rapid Rate 


\subsection{UDM Circuit}

The UDM circuit senses when the device pointer is grounded and the shapes are being drawn. The complete working of UDM is explained under the section 3.2 of the paper. This circuit has been placed just beside the pointer on the bottom surface of the horizontal circuit.

\subsection{Vertical Circuit (VC)}

The reason that this circuit has been named as ' $\mathrm{VC}$ ' is that, the circuit is placed inside a vertical cylinder that is fitted over the horizontal box. The vertical contains following components;
A. Finger Button (FB)
B. Left Button (LB)
C. $7 \mathrm{C}$ Capacitor $(7 \mathrm{C})$
D. Middle Button (MB)
E. Two-Way Switch (TWS)
F. VC Capacitor (VC)
G. LED with Resistor (LED)
H. Scrolling Device (SD)

\section{CONCLUSION}

RaTTy is very useful and affordable product. This may be widely used in many areas. The really neat thing about a digital pen is what happens to the information it captures after it's sent to your computer.

The main purpose of making this digital pen is to enable an ordinary user to make their writing comfortable. They can write in their own ordinary style on a normal paper and this would save the written data in digital format as .doc.

In the coming time, the mouse can be built in this particular shape that would appear as a pen and work like digital pen as well as computer mouse. Hence this would become really comfortable for a user to interact with the computer and write digitally.

\section{ACKNOWLEDGEMENTS}

We would like to express our deep sense of gratitude and convey thanks to everyone including experts, family members and friends who helped us and supported to take the responsibility of working on such a complicated research work. Our thanks to the experts who have contributed towards development of the device. We would like to give special thanks to Mr. Divyanshu Tiwari for helping in circuit designing.

\section{REFERENCES}

[1] Avago Technologies "Optical Mice \& How They Work"

[2] Avago Technologies Announces Miniature Laser Navigation Sensors for Mouse Applications". January 28 2008. Retrieved 2013-03-25.

[3] Tavernier, Karel. "PCB Fabrication Data - A Guide". Ucamco. Retrieved 8 January 2015.

[4] The Livescribe Platform (commercial whitepaper)

[5] Shelly, Gary B.; Misty E. Vermaat (2009). Discovering Computers: Fundamentals. Cengage Learning. ISBN 978-0-495-80638-7. Retrieved 3 November 2009.

[6] John Markoff (May 10, 1982). "Computer mice are scurrying out of R\&D labs". InfoWorld 4 (18): 10-11. ISSN 0199-6649.

[7] Sol Sherr (1988). Input Devices. Academic Press.

[8] "Circuit Symbols". electronicsclub.info. Retrieved 2 August 2014

[9] Royden, C. S.; Moore, K. D. (2012). "Use of speed cues in the detection of moving objects by moving observers". Vision Research 